Sonnleitner, Thomas, Turton, David A., Waselikowski, Stefan, Hunger, Johannes, Stoppa, Alexander, Walther, Markus, Wynne, Klaas, and Buchner, Richard (2014) Dynamics of RTILs: a comparative dielectric and OKE study. Journal of Molecular Liquids, 192 . pp. 19-25. ISSN 0167-7322

Copyright (C 2013 Elsevier B.V.

http://eprints.gla.ac.uk/93249/

Deposited on: 29 April 2014

Enlighten - Research publications by members of the University of Glasgow http://eprints.gla.ac.uk 


\title{
Dynamics of RTILs: A Comparative Dielectric and OKE study
}

\author{
Thomas Sonnleitner $^{\mathrm{a}}$, David A. Turton ${ }^{\mathrm{b}}$, Stefan Waselikowski ${ }^{\mathrm{c}}$, Johannes Hunger ${ }^{d}$, Alexander Stoppa ${ }^{\mathrm{a}}$, Markus Walther ${ }^{\mathrm{c}}, \mathrm{Klaas}$ \\ Wynne $^{\text {b }}$, Richard Buchner ${ }^{\mathrm{a}}$ \\ ${ }^{a}$ Institut für Physikalische and Theoretische Chemie, Universität Regensburg, 93040 Regensburg, Germany \\ ${ }^{\mathrm{b}}$ School of Chemistry and WestCHEM, University of Glasgow, G12 8QQ, UK \\ ${ }^{\mathrm{c}}$ Freiburger Materialforschungszentrum, Albert-Ludwigs-Universität Freiburg, 79104 Freiburg, Germany \\ ${ }^{\mathrm{d}}$ Max Planck Institute for Polymer Research, 55128 Mainz, Germany
}

\begin{abstract}
The dynamics of room-temperature ionic liquids (RTILs) were studied by investigating their dielectric relaxation (DR) and timeresolved optical Kerr-effect (OKE) spectra in the frequency range of $\sim 10 \mathrm{MHz}$ to $\sim 20 \mathrm{THz}$. For the studied RTILs the OKE and DR spectra are dominated by a relaxation in the GHz region and extend to a relatively sharp band at around $10 \mathrm{THz}$. Whilst the first feature is mainly associated with the structural relaxation of the fluid through ion rotation ( $\alpha$ relaxation), the second indicates the short-time limit of intermolecular dynamics. The rather featureless intermediate region is mainly associated with intermolecular vibrations that are strongly coupled to hindered rotations. In contrast to other RTILs, imidazolium salts show an additional sub- $\alpha$ relaxation which dominates the OKE signal and is indicative of the breathing motion of rather long-lived cages.

Mixed with polar solvents RTILs were found to retain their ionic liquid-like character up to relatively high levels of dilution, but with the overall dynamics considerably speeded up. Below RTIL mole fractions of $\sim 0.2-0.4$ these systems behave like conventional electrolyte solutions with more or less pronounced ion pairing.
\end{abstract}

Keywords: Dielectric relaxation, ultra-fast optical Kerr-effect, cooperative dynamics, spectroscopy, room-temperature ionic liquids

\section{Introduction}

Room-temperature ionic liquids (RTILs) are often described as designer solvents due to their almost unlimited number of cation-anion combinations, allowing the fine-tuning of their properties with respect to specific applications [1, 2]. Among the class of aprotic RTILs imidazolium salts are undoubtedly the most intensively studied sub-class but salts composed of sulfonium, pyrrolidinium, pyridinium, phosphonium and ammonium cations are increasingly attracting interest. Due to their negligible volatility, high conductivity and generally large electrochemical window, aprotic RTILs are industrially interesting as reaction media and for potential applications in separation technology [3, 4]. Recently, the class of protic ionic liquids (PILs), obtained by proton transfer between a Brønsted acid and a Brønsted base, became of particular interest as possible replacements for aqueous solutions in batteries and fuel cells [5]. Naturally, the wide range of actual and potential applications for RTILs has inspired an impressive range of research activity $[3,4,5,6]$. Nevertheless, many fundamental issues concerning the structure and especially the dynamics of these fascinating fluids are still not well understood. The main goal of DFG pri-

\footnotetext{
${ }^{*}$ Corresponding author

Email address: Richard.Buchner@chemie.uni-regensburg.de (Richard Buchner ${ }^{\mathrm{a}}$ )
}

ority program SPP 1191 was fostering investigations into fundamental physico-chemical properties of RTILs and their mixtures with other components. The present contribution summarizes our investigations of the collective dynamics of such systems within SPP 1191.

Around room temperature, the timescale of the relevant rotational and translational motions of RTILs spans from a few hundred femtoseconds to several nanoseconds, roughly corresponding to frequencies between $\sim 10 \mathrm{THz}$ and $\sim 10 \mathrm{MHz}$. This dynamic range was covered by broadband dielectric relaxation (DR) spectroscopy, which is sensitive to fluctuations of the macroscopic dipole moment of the sample [7]. This technique is especially suited to the investigation of RTILs and their mixtures as it not only covers the entire frequency range required (albeit with several instruments, see below) but also because the cations and some of the anions generally constituting RTILs possess a permanent dipole moment. In addition, DR spectroscopy is the only technique available at present to directly determine the static relative permittivity (dielectric constant), $\varepsilon$, of conducting samples, yielding thus a measure for RTIL polarity [2, 8, 9]. Our dielectric studies were complemented by ultra-fast optical Kerr-effect (OKE) spectroscopy, which can cover the same frequency range and is sensitive to fluctuations of the polarizability anisotropy [10]. As DR and OKE spectroscopy can be viewed as the low-frequency counterparts of, 
respectively, infrared and Raman spectroscopy, the techniques are to some extent complementary $[11,12]$. In the following we will focus on RTIL dynamics, with an excursion to RTIL+polar solvent mixtures. For space reasons only an overview of general trends will be given. For details we refer to the literature and forthcoming publications of our group. Although the static permittivity is directly measured, for the sake of brevity we abstain from a discussion of this quantity and refer to the contribution of H. Weingärtner et al. to this Special Issue. For a discussion of the long-time evolution of RTIL dynamics (relaxation times $\gtrsim 10^{-6} \mathrm{~s}$ ), dominated by charge-transport phenomena and (at sufficiently low temperature) glass formation, the reader is referred to the contribution of F. Kremer et al. to this Special Issue and some recent publications [13, 14, 15, 16, 17].

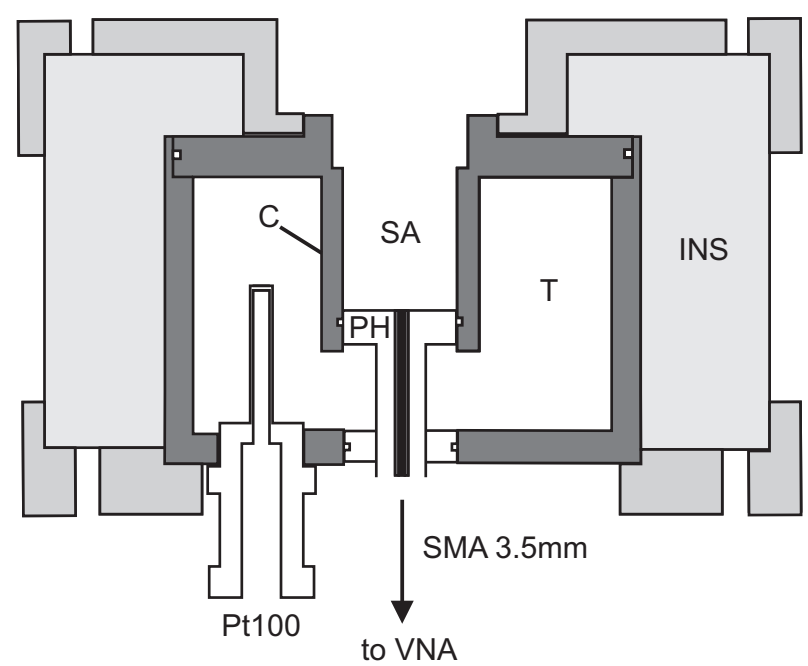

Figure 1: Measurement cell for the Agilent $85070 \mathrm{E}-020$ probe (similar for Agilent 85070E-050): (PH) probe head; (C) Cell body (carbon-filled PTFE); (SA) sample volume; (T) thermostat jacket connected to external thermostat; (INS) insulation; (Pt100) temperature sensor. The headspace above the sample can be kept under dry $\mathrm{N}_{2}$ atmosphere (details not shown).

\section{Experimental Techniques}

\subsection{Dielectric Relaxation Spectroscopy}

DR spectroscopy measures the linear response of the macroscopic polarization of a sample to an external time-dependent electric field $[18,19]$ which can be expressed as the derivative over time of the time-correlation function $[10,18]$ of the macroscopic dipole moment, $\vec{M}$,

$$
S_{\mathrm{DRS}}(t) \propto \frac{1}{k_{\mathrm{B}} T} \frac{\mathrm{d}}{\mathrm{d} t}\langle\vec{M}(t) \cdot \vec{M}(0)\rangle
$$

In the frequency domain, the experimentally accessible quantity is the generalized complex permittivity

$$
\hat{\eta}(v)=\hat{\varepsilon}(v)-\mathrm{i} \kappa /\left(2 \pi v \varepsilon_{0}\right)
$$

at frequency, $v$, where the second term of Eq. (2) represents the Ohmic loss caused by diffusive charge transport with dc conductivity, $\kappa ; \varepsilon_{0}$ is the permittivity of free space. The first term, the complex permittivity $\hat{\varepsilon}(v)=\varepsilon^{\prime}(v)-\mathrm{i} \varepsilon^{\prime \prime}(v)$ with relative permittivity, $\varepsilon^{\prime}(v)$, and dielectric loss, $\varepsilon^{\prime \prime}(v)$, contains all frequency-dependent contributions to polarization. For the present systems these mainly arise from dipole rotation and libration, as well as from hindered translations (frequencydependent conductivity) and inter- and intramolecular vibrations, reflecting thus the entire dynamics of the studied liquid.

To accomplish the required coverage of several orders of magnitude in frequency in a region still difficult to access, several instruments were required. The lower-frequency region up to $89 \mathrm{GHz}$ was measured in Regensburg using a waveguide interferometer for the frequency range of $60 \leq v / \mathrm{GHz} \leq 89$ $[20,21]$ and a vector network analyzer (VNA, Agilent E8364B) with electronic calibration module (ECal, Agilent N4693A) in reflection mode for $0.01 \leq v / \mathrm{GHz} \leq 50$. The VNA was operated with home-made coaxial-line cut-off cells of varying cell constants suitable for the range $0.01-1 \mathrm{GHz}$ [22], as well as two commercial open-ended coaxial-line probes covering 0.2-20 GHz (Agilent 85070E-020) and $\sim 5-50 \mathrm{GHz}$ (Agilent $85070 \mathrm{E}-050$ ). The probes (PH of Fig. 1) were mounted in cells matching their outer diameter and made of carbon-filled PTFE to suppress unwanted waveguide modes. The cells were inserted in a thermostat jacket (T) equipped with a Pt100 temperature sensor, enabling measurements with a temperature uncertainty of $\pm 0.03^{\circ} \mathrm{C}$ in the range of -35 to $90{ }^{\circ} \mathrm{C}$. The calibration procedure for the reflection experiments has been described previously [22, 23, 24]. Additional VNA measurements were made for selected samples using variable path-length waveguide transmission cells [20] similar to that of the interferometer but ranging from $8.5 \leq v / \mathrm{GHz} \leq 40$. Since such measurements do not require calibration, albeit at the cost of a large sample volume, their results can be used to crosscheck the data obtained from the open-ended coaxial-line probes.

The intermediate frequency range of $0.2 \lesssim v / \mathrm{THz} \leq 2.5$ was measured in Freiburg using time-domain $\mathrm{THz}$ spectroscopy $[25,26]$, which also measures the complex dielectric spectrum via the absorption coefficient and refractive index of the sample. Figure 2 shows the schemes of the reflection $(0.08 \leq v / \mathrm{THz} \leq$ $0.8)$ and transmission $(0.2 \leq v / \mathrm{THz} \leq 2.5)$ setups and the corresponding cells.

In addition to the DR and terahertz measurements, farinfrared absorption spectra (Bruker Vertex 70) were measured for $0.6-20 \mathrm{THz}$ with the liquids held between a pair of polymethylpentene (TPX) windows [12]. After concatenating the absorption coefficient spectra of all three laboratories, the refractive indices for the far-infrared region were derived by Kramers-Kronig transformation [18], so that the total permittivity spectrum, $\hat{\eta}(v)$, between $\sim 10 \mathrm{MHz}$ and $\sim 20 \mathrm{THz}$ was available. $^{1}$

\subsection{Optical Kerr-Effect Spectroscopy}

OKE spectroscopy measures the derivative over time of the two-point time-correlation function of the anisotropic part of

\footnotetext{
${ }^{1}$ Note that due to excessive loss arising from the high dc conductivity of most RTILs the accessible minimum frequency, $v_{\min }$, for $\hat{\varepsilon}(v)$ varies with $\kappa$ as sensible determination of $\varepsilon^{\prime \prime}$ requires that its magnitude exceeds the experimental uncertainty of the total loss, $\eta^{\prime \prime}\left(v_{\min }\right)$.
} 

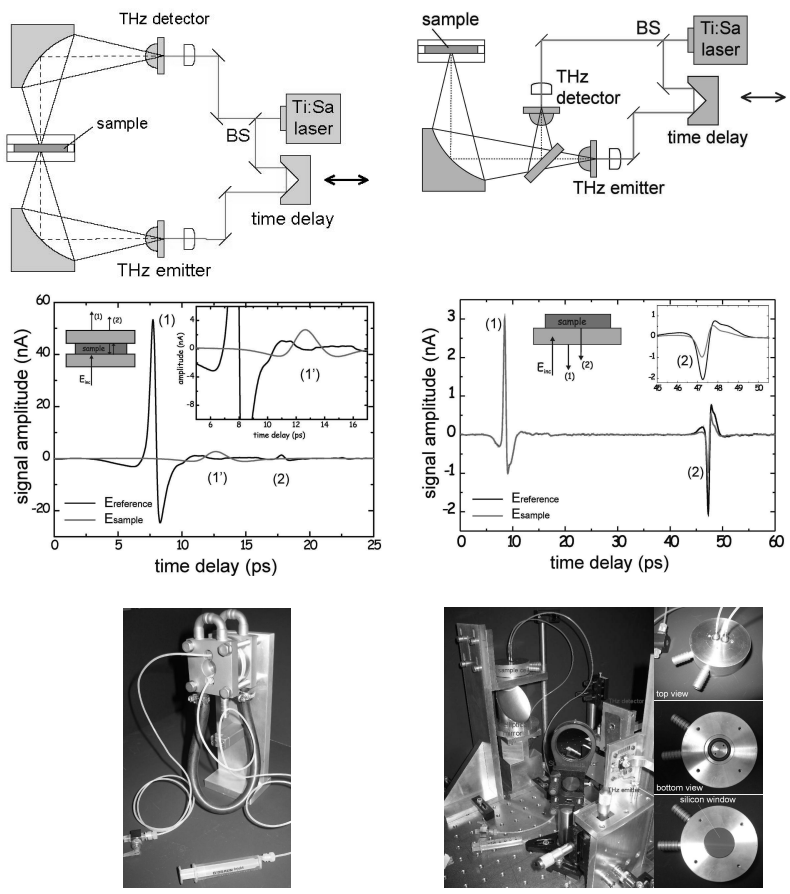

Figure 2: Instrument configuration, measured signals and sample holders for time-domain $\mathrm{THz}$ spectroscopy in transmission (left) and reflection (right) mode at Freiburg.

the many-body polarizability tensor, $\Pi_{x y}$,

$$
S_{\mathrm{OKE}}(t) \propto \frac{1}{k_{\mathrm{B}} T} \frac{\mathrm{d}}{\mathrm{d} t}\left\langle\Pi_{x y}(t) \Pi_{x y}(0)\right\rangle
$$

It is therefore - like DR spectroscopy - primarily sensitive to rotational motions and only weakly to translations. Although both techniques are complementary for intramolecular modes, this is not necessarily the case at low frequencies where strong interactions are involved. Thus, although the observed modes may arise from different molecular moieties, the same dynamics are generally measured with different intensities for the two techniques [12].

To capture the wide time-span of intermolecular dynamics, two OKE set-ups were employed. The faster dynamics were measured using a $7 \mathrm{~nJ}, 20 \mathrm{fs}$ pulse (Coherent Mira-SEED) [27] with weaker relaxations at longer times recorded using a regeneratively-amplified setup (Coherent Mantis Legend USX) with a $1 \mu \mathrm{J}$ pulse stretched to a duration of $\sim 1 \mathrm{ps}$ [28].

The spectra obtained with both techniques were fitted (Igor, Wavemetrics) in the frequency-domain with an in-house developed software [12, 29] using standard and specialized empirical functions to model relaxation and resonance processes as described previously [12, 29, 30].

\subsection{Auxiliary Measurements}

Densities, $\rho$, were measured by a vibrating-tube densimeter (Anton Paar, Graz, Austria, DMA $5000 \mathrm{M}$ ) yielding $\rho$ with a nominal uncertainty of $\pm 5 \cdot 10^{-6} \mathrm{~g} \mathrm{~cm}^{-3}$ in the temperature range $5-85^{\circ} \mathrm{C}$. Viscosities, $\eta$, were measured with a rolling ball microviscometer (Anton Paar, Graz, Austria, AMVn) with $\sim 0.5 \%$ repeatability in the range of 5 to $125^{\circ} \mathrm{C}$. Electrical conductivities, $\kappa$, were determined in the temperature range of -45 to $190{ }^{\circ} \mathrm{C}$ with an overall uncertainty of $0.5 \%$ using the two setups described previously [31, 32].

\section{Dynamics of Aprotic RTILs}

\subsection{General Remarks}

Among the sub-class of aprotic RTILs, imidazolium salts are most intensively studied and widely considered as representative ionic liquids. Within the framework of the present investigation, a number of RTILs composed of 1-alkyl-3methylimidazolium cations, $\left[\mathrm{C}_{n} \mathrm{mim}\right]^{+}$with $n=2,4,6$, and anions such as $[\mathrm{DCA}]^{-},\left[\mathrm{NTf}_{2}\right]^{-},\left[\mathrm{BF}_{4}\right]^{-},\left[\mathrm{PF}_{6}\right]^{-},[\mathrm{OAc}]^{-}$, $\left[\mathrm{TfO}^{-},\left[\mathrm{EtSO}_{4}\right]^{-},[\mathrm{FSA}]^{-}\right.$, and $[\mathrm{BETI}]^{-}$, was investigated either only by DR spectroscopy (generally over a limited frequency range) or jointly by DR and OKE spectroscopy over the entire range. Additionally, a selection RTILs with pyridinium, pyrrolidinium, and sulfonium cations has been jointly characterized. Already published material can be found in Refs. $[12,33,34,35,36,37,38,39]$. From a review of these results the general characteristics discussed below can be observed.
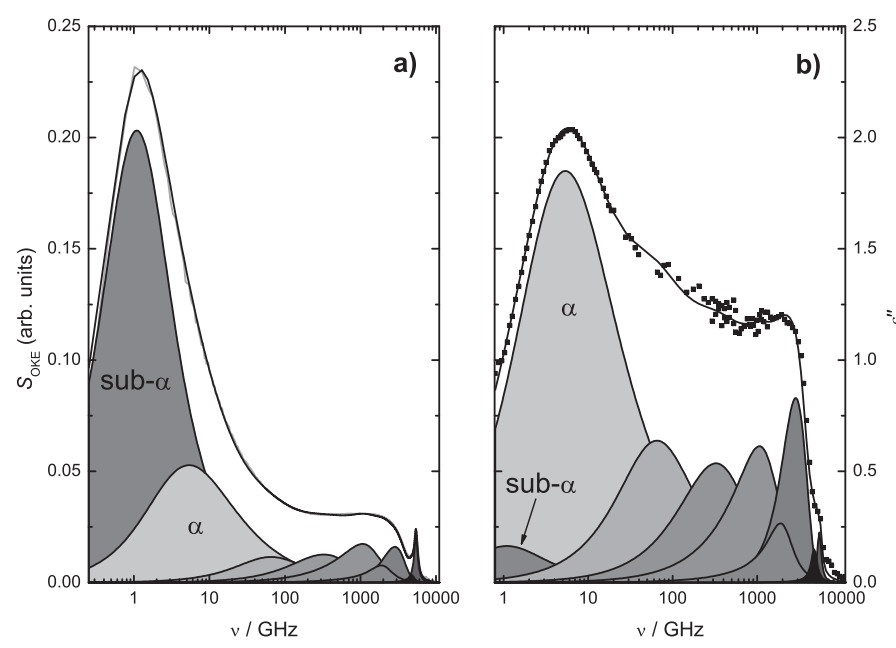

Figure 3: Global fit of (a) OKE (imaginary part) and (b) DR (dielectric loss) spectra of 1-ethyl-3-methylimidazolium dicyanamide, [ $\left.\mathrm{C}_{2} \mathrm{mim}\right][\mathrm{DCA}]$, at $25^{\circ} \mathrm{C}$. Gray solid line in (a) and full symbols in (b) are experimental data; black solid lines are the fits and shaded areas represent the individual component fit functions [12].

At first glance, the DR and OKE spectra of all the studied aprotic RTILs are similar with respect to their overall shape (Figures $3 \& 4$ ). Both are dominated by a mode at low frequencies (typically around $0.5-5 \mathrm{GHz}$ ), a more or less featureless intermediate region, and a pronounced cutoff at $\sim 8 \mathrm{THz}$. These spectra can be decomposed into a series of relaxation modes at lower frequencies $(\lesssim 50 \mathrm{GHz})$ and rather broad (strongly damped) resonance modes in the $\mathrm{THz}$ region, all of which are characteristic for the intermolecular dynamics. Sharper peaks in the $\mathrm{THz}$ region are due to intramolecular vibrations.

However, a closer look reveals a marked difference between the spectra of imidazolium salts and those of pyridinium-, pyrrolidinium-, and sulfonium-RTILs. The combined fitting 
of both DR and OKE spectra showed that the dominant lowfrequency peak of imidazolium ILs is a composite and can be split up into two separate processes labeled as $s u b-\alpha$ and $\alpha$ relaxation respectively, with peak positions $v_{s u b-\alpha}^{\mathrm{pk}}<v_{\alpha}^{\mathrm{pk}}$, i.e. relaxation times $\tau_{s u b-\alpha}>\tau_{\alpha}$. Whilst the sub- $\alpha$ mode largely dominates the OKE signal it is hardly discernible in the dielectric spectrum (Figure 3) [12, 37]. In marked contrast, the sub$\alpha$ relaxation cannot be detected the spectra of pyrrolidinium-, pyridinium- and sulfonium-RTILs. Here only the $\alpha$ mode is found with both methods (e.g. Figure 4).

\section{2. sub- $\alpha$ Relaxation}

The $s u b-\alpha$ relaxation is unique to imidazolium RTILs where it dominates the OKE spectra [12, 37] (Figure 3). Sofar it has not been observed for other cations. This contribution is very weak in the DR spectra and without low-frequency Kerr-effect data it would almost certainly have been overlooked. The $s u b-\alpha$ mode implies large fluctuations of the polarizability anisotropy that hardly affect the macroscopic dipole moment, i.e. does not involve major changes in the orientation of the cation (and, where present, anion) dipole moments. This points to a breathing mode of mesoscopic aggregates essentially normal to the average dipole orientation of the cluster. Aggregates of $\pi$ stacked cations and their surrounding anions are reasonable candidates compatible with MD simulations [40, 41, 42] and scattering experiments [43]. It should be noted that the lifetime of the caging effects observed in MD simulations of RTIL diffusion, see $e . g$. Ref. [44], as well as the charge-hopping times deduced from low-frequency dielectric measurements [45, 46, 17] are comparable to the $s u b-\alpha$ relaxation time, which is in the order of a few hundred picoseconds at room temperature.
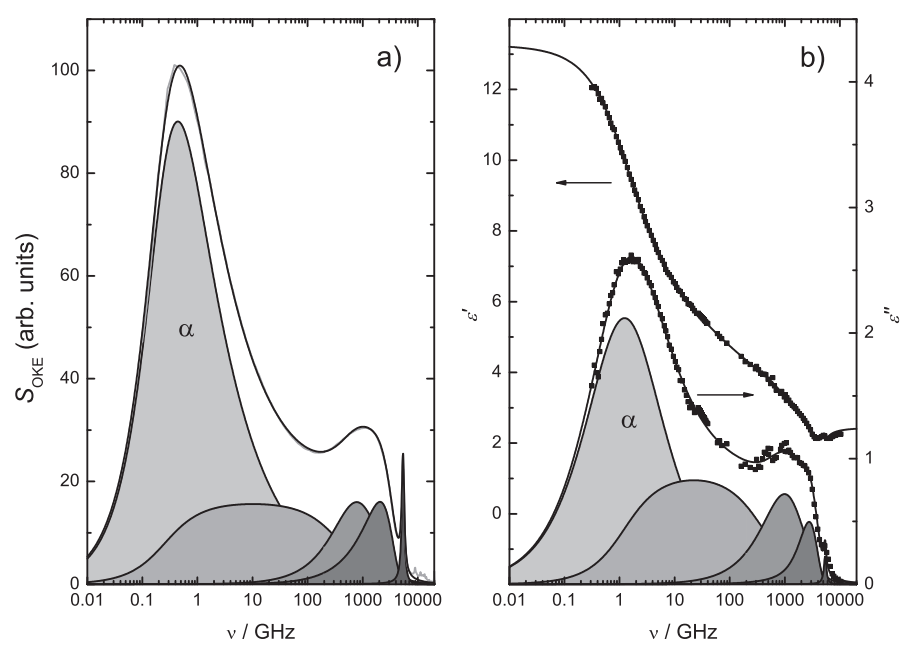

Figure 4: Global fit of (a) OKE and (b) DR spectra of 1-butyl-1methylpyrrolidinium dicyanamide, $\left[\mathrm{P}_{14}\right][\mathrm{DCA}]$, at $5{ }^{\circ} \mathrm{C}$. Gray solid line in (a) and full symbols in (b) are experimental data; black solid lines are the global fits and shaded areas represent the individual fit functions.

\section{3. $\alpha$ Relaxation}

At room temperature, the $\alpha$ relaxation dominating the DR response (Figures $3 \& 4$ ) peaks in the $0.7-5 \mathrm{GHz}$ region. Quan- titative evaluation of its amplitude, $S_{\alpha}^{\text {DRS }}$, revealed that for imidazolium salts the reorientation of dipolar $\left[\mathrm{C}_{n} \mathrm{mim}\right]^{+}$cations provides the major contribution $[34,35]$. Cation rotation is also the main contributor to the OKE $\alpha$ mode [12]. Dipolar anions give rise to an additional relaxation at somewhat higher frequencies. Generally, the amplitude of this mode is considerably smaller than expected from the dipole moment of the anion, whereas the value of $S_{\alpha}^{\mathrm{DRS}}$ is increased compared to the corresponding RTIL with non-polar anion. This reveals strong orientational correlations of cation and anion dipole moments in such RTILs, with a significant fraction of the anions slaved in their motions to cation dynamics [36]. We are still working on the detailed evaluation of our data for non-imidazolium RTILs but it appears that, depending on their structure, both cations and anions contribute to the $\alpha$ mode but with different relative weights for DR and OKE. Common to all RTILs is that the $\alpha$ peak is rather broad, suggesting large dynamic heterogeneity.

Simulations suggest that at least for imidazolium RTILs the $\alpha$ relaxation is not a pure rotational motion but also significantly involves translations of cations and anions [47]. This might explain why the effective volumes of rotation derived from a Stoke-Einstein-Debye analysis of $\tau_{\alpha}$ yields values incompatible with cation reorientation via rotational diffusion [35]. Indeed by comparing the $\tau_{\alpha}$ values from DR and OKE it can be shown that $\left[\mathrm{C}_{n} \mathrm{mim}\right]^{+}$cations reorient through large angle jumps [12], in line with the simulation results of Shim et al. [48]. One could speculate that the large-scale cooperative motions giving rise to the $s u b-\alpha$ mode prepare the triggering event that leads to cation flip.

\subsection{Short-time Dynamics}

The high complexity of RTIL dynamics becomes apparent in the OKE and DR spectra by the flat but rather large contribution above $\sim 50 \mathrm{GHz}$. Intramolecular vibrations are generally weak at such low frequencies. Close inspection revealed ion-specific features at $v \gtrsim 1 \mathrm{THz}\left(30 \mathrm{~cm}^{-1}\right)$ to be present both in DR and OKE spectra $[11,12]$. This region has been intensively studied by OKE during the last decade, see Refs. [10, 49, 50, 51, 52] and literature cited therein. A pronounced peak at $1-3 \mathrm{THz}$ is a general characteristic of liquids and well established as the librational motion, i.e. the hindered rotation of (here) the ions in their fluctuating cage of surrounding neighbors. The apparent high-frequency cutoff marks the short-time limit ( $\sim 100 \mathrm{fs})$ of these motions. The frequency of the libration mode is determined by the ion's moment of inertia as well as the potential well of the cage. Generally, anion librations occur at lower frequencies $(\sim 1 \mathrm{THz})$ than cation librations $(\sim 2-5 \mathrm{THz})$ because of the lower restoring force for the first [52]. Although there is only a limited number of dielectric measurements for RTILs in this region so far, these appear to observe the same processes as OKE spectroscopy.

The flat intermediate frequency region of $\sim 0.05-1 \mathrm{THz}$ can be fitted by a sum of of resonance modes [11] (Figure 3). However, in the light of new results it seems more appropriate to use a so-called "constant-loss" term for this region (Figure 4) consistent with studies of other strongly interacting liquids [28]. This mainly translational mode is associated with the rattling of 
molecules in their cage of surrounding neighbors superimposed on the formation and decay of these cages. An argument from MD simulations if favor of such an assignment also for RTILs is provided by the strong collective translational contributions (interionic vibrations) to the simulated dielectric spectrum in this frequency range $[47,53]$.

Table 1: Arrhenius activation energies, $E_{\mathrm{A}}$, in $\mathrm{kJ} \mathrm{mol}^{-1}$, derived from the $\alpha$ relaxation of DR and OKE, and from viscosity, $\eta$, and conductivity, $\kappa$.

\begin{tabular}{lcccc}
\hline RTIL & $E_{\mathrm{A}}^{\mathrm{DRS}}$ & $E_{\mathrm{A}}^{\mathrm{OKE}}$ & $E_{\mathrm{A}}^{\kappa}$ & $E_{\mathrm{A}}^{\eta}$ \\
\hline EAN & $21.7 \pm 0.3$ & $23.9 \pm 0.6$ & $23.1 \pm 0.6$ & $26.2 \pm 0.7$ \\
$\mathrm{PAN}$ & $27.0 \pm 1.1$ & $25.5 \pm 0.3$ & - & $30.4 \pm 0.7$ \\
{$\left[\mathrm{~S}_{222}\right]\left[\mathrm{NTf}_{2}\right]$} & $19.9 \pm 1.1$ & $20.9 \pm 1.7$ & $26.5 \pm 0.8$ & $28.7 \pm 0.8$ \\
{$\left[\mathrm{P}_{14}\right][\mathrm{DCA}]$} & $18.2 \pm 1.6$ & $23.4 \pm 0.3$ & $25.2 \pm 0.6$ & $27.4 \pm 0.5$ \\
{$\left[\mathrm{C}_{2}\right.$ mim $]\left[\mathrm{NTf}_{2}\right]$} & $19.8 \pm 1.0$ & - & $25.1 \pm 0.7$ & $27.1 \pm 0.6$ \\
\hline
\end{tabular}
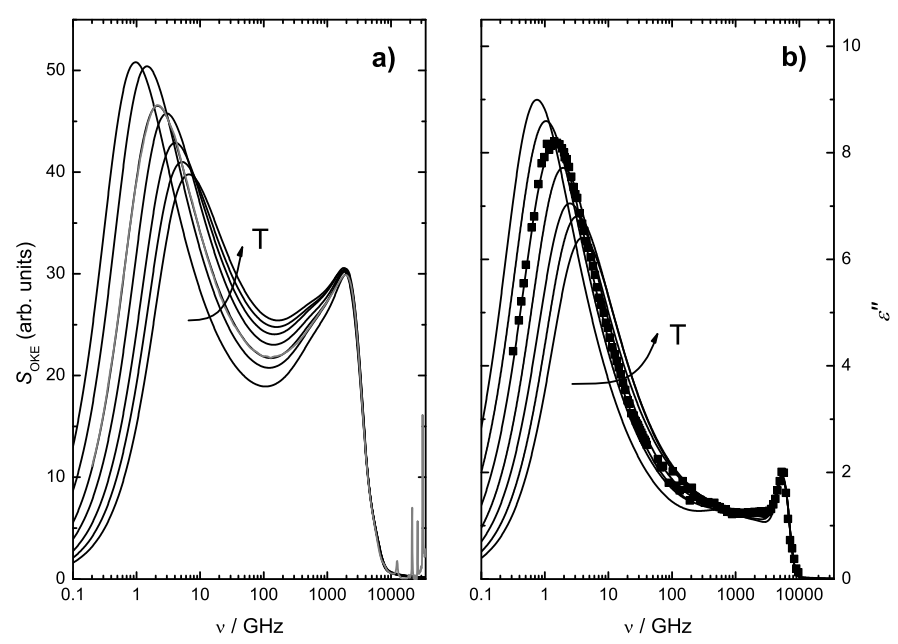

Figure 5: (a) Optical Kerr-effect and (b) dielectric spectra from $200 \mathrm{MHz}$ to $20 \mathrm{THz}$ between $5^{\circ} \mathrm{C}$ and $65^{\circ} \mathrm{C}$ for ethylammonium nitrate (EAN). Full symbols are experimental data at $25^{\circ} \mathrm{C}$ and solid lines are the global fits [28].

\section{Dynamics of Protic RTILs}

Since protic RTILs are gaining more and more interest recently [5] and only limited information on their cooperative dynamics is available $[54,55,56]$ it seemed worthwhile to investigate ethylammonium nitrate (EAN) and propylammonium nitrate (PAN) as typical representatives of this RTIL sub-class $[28,57]$. A main feature of protic RTILs is their ability to form strong inter-ionic hydrogen bonds and it is expected that this extended $\mathrm{H}$-bond network is reflected in the dynamics. Since $\mathrm{NO}_{3}^{-}$ has no dipole moment, the DR signal is essentially dominated by cation motions whereas the OKE response is dominated by the dynamics of the highly polarizable anion, i.e. for EAN and PAN these techniques monitor different species.

The DR and OKE spectra of EAN (Figure 5) and PAN resemble those of aprotic non-imidazolium RTILs in their overall shape (Figure 4). However, there is no indication of a $s u b-\alpha$ mode. The most notable difference are the pronounced peaks at $\sim 2 \mathrm{THz}$ in the OKE and $\sim 5.5 \mathrm{THz}$ in the DR spectrum. Based on cluster calculations, Fumino et al. [55] suggested that these two strong bands are equivalent to the hydrogen bond bend and stretch of water. However, the librational bands of water are observed in the $400-800 \mathrm{~cm}^{-1}$ region (due to the low moment of inertia of the $\mathrm{H}_{2} \mathrm{O}$ molecule) whereas for nitrate and ethylammonium ions these should appear below $300 \mathrm{~cm}^{-1}(10 \mathrm{THz})$. Keeping in mind that the dielectric and OKE signals are both primarily sensitive to rotations, it therefore appears that the observed peaks at $\sim 2 \mathrm{THz}$ and $\sim 5.5 \mathrm{THz}$ are librational. This does not argue against extensive hydrogen bonding in these liquids - we can even expect that hydrogen bonding will affect the restoring forces of cation and anion libration and thus determine their positions - but from the involved ion masses we think that the shoulder observed just below $1 \mathrm{THz}$ (in the OKE spectra, Figure 5(a)) would be a more likely candidate for the hydrogen bond stretch.

An indication for extensive hydrogen bonding, leading to strong cooperativity in the motions of anions and cations is the Arrhenius activation energy, $E_{\mathrm{A}}$ of the $\alpha$ relaxation. ${ }^{2}$ As can be seen from Table 1 the $E_{\mathrm{A}}$ values for the cation (probed by $\mathrm{DR}$ ) and anion (OKE) reorientation as well as for conductivity are very similar for EAN. This also applies to ion rotation in PAN (where $E_{\mathrm{A}}^{K}$ remains to be determined). For aprotic RTILs, which do not form an extended hydrogen bond network, the activation energies for the $\alpha$ relaxation seem to be consistently smaller than those of EAN and PAN. They are also smaller than the corresponding $E_{\mathrm{A}}^{\kappa}$ values. More direct evidence comes from a comparison of DR and time-resolved infrared spectra showing that the reorientation of the entire cation and of the $\mathrm{N}-\mathrm{H}$ bond vector have identical activation energy and occur through jumps of $\sim 106^{\circ}[57]$.

\section{RTILs in Polar Solvents}

Several imidazolium salts were also studied by DR spectroscopy in mixtures with polar solvents, albeit only in the frequency range $0.2 \leq v / \mathrm{GHz} \leq 89$. Mixtures of $\left[\mathrm{C}_{n} \mathrm{mim}\right]\left[\mathrm{BF}_{4}\right]$ (with $n=2,4,6$ ) [60] and $\left[\mathrm{C}_{6} \mathrm{mim}\right]\left[\mathrm{NTf}_{2}\right]$ (Figure 6) in Acetonitrile $(\mathrm{AN})$, or of $\left[\mathrm{C}_{4} \mathrm{mim}\right]\left[\mathrm{BF}_{4}\right][61]$ and $\left[\mathrm{C}_{2} \mathrm{mim}\right]\left[\mathrm{EtSO}_{4}\right]$ [36] in dichloromethane exhibit bimodal spectra. It turns out that both are composites with the high-frequency mode arising from solvent reorientation and (to a minor extent) fast RTIL contributions. The contribution at lower frequencies is dominated by cation reorientation but for RTIL mole fractions $x \lesssim 0.3$ also contact ion pairs (CIPs) contribute. For acetonitrile mixtures, a contribution from bound solvent molecules can also be detected (Figure 6). From the detailed analysis of the relaxation amplitudes a cation solvation number of $\sim 7$ was derived

\footnotetext{
${ }^{2}$ Note that most RTILs easily supercool and, as expected, their dynamic properties generally follow the Vogel-Fulcher-Tammann relation when observed over a sufficiently large temperature range [17, 31, 58, 59]. Thus, the $E_{\mathrm{A}}$ values of Table 1, determined for the temperature range of the present DR measurements (278.15-338.15 K) where an Arrhenius equation was sufficient to fit the data, have to be regarded as apparent activation energies at ambient temperature.
} 
for $\left[\mathrm{C}_{n} \operatorname{mim}\right]\left[\mathrm{BF}_{4}\right](n=2,4,6)+$ acetonitrile at $x \rightarrow 0$ [60]. Since this solvation number is independent of the cation's alkyl chain we may conclude that these bound acetonitrile molecules essentially interact with the imidazolium ring.

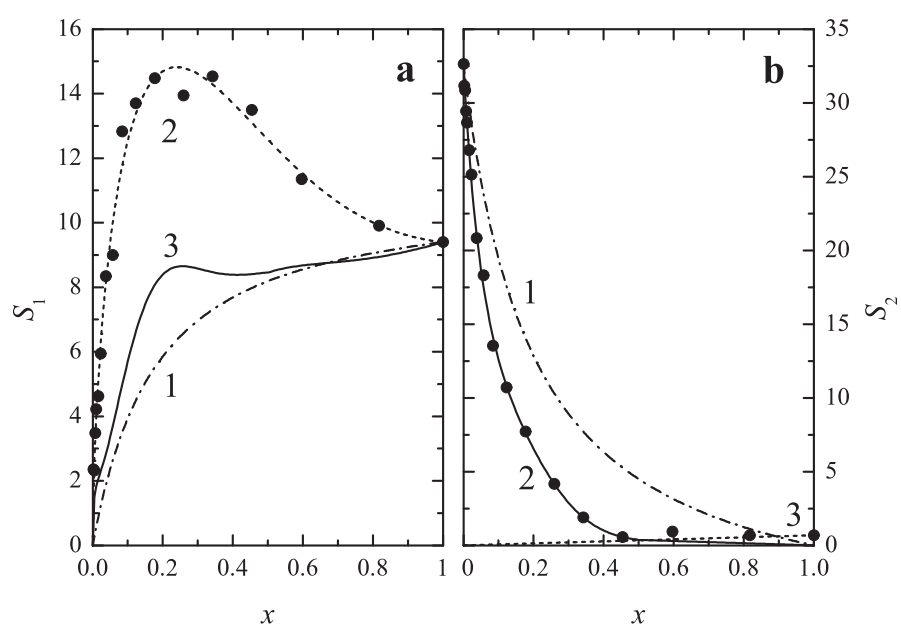

Figure 6: Relaxation amplitudes $S_{1}$ and $S_{2}$ of $\left[\mathrm{C}_{6}\right.$ mim] $\left[\mathrm{NTf}_{2}\right]+$ acetontitrile mixtures at $25^{\circ} \mathrm{C}$. For the IL-dominated low-frequency mode $S_{1}$ (a), curve 1 shows the expected IL contribution, 2 a fit of the experimental data $(\bullet)$, and 3 the experimental results corrected for the contribution of bound AN. For the AN-dominated fast mode (b), curve 1 shows the expected AN contribution, 2 a fit of the experimental data $(\bullet)$, and 3 the assumed IL contribution (see Ref. [60] for details).

A general feature of the studied mixtures is that although their dynamics gets considerably faster and more homogeneous on dilution their overall character strongly resembles that of the pure RTIL even down to $x \approx 0.2-0.4$. Thus, in this low-dilution region the added solvent "lubricates" the RTIL but its moltensalt like character is retained. On further dilution a smooth transition to the behavior of concentrated electrolyte solutions is observed before long-lived contact ion pairs typical for moderately concentrated electrolyte solutions appear at $x \lesssim 0.1$ $[36,61,60]$. As expected, the degree of ion pairing is strongly dependent on solvent polarity but is always comparable to association constants of common salts with organic cations in these solvents $[62,63]$.

\section{Concluding remarks}

The collective dynamics of RTILs were studied by two complementary spectroscopic techniques, with DR spectroscopy probing the dipole vector and OKE spectroscopy being sensitive to the polarizability anisotropy of the sample. For all studied RTIL classes DR and OKE spectra were similar in overall shape, with a dominating relaxation roughly around $1 \mathrm{GHz}$, an intermediate "constant-loss" region, and more or less pronounced libration bands at $\sim 1-5 \mathrm{THz}$ followed by a pronounced cut-off at $\sim 10 \mathrm{THz}$. In the $\mathrm{THz}$ region intermolecular dynamics may overlap with intramolecular vibrations.

The large bandwidth covered by RTIL spectra indicated that for these liquids the timescale of intermolecular motions ranges from $\sim 100$ femtoseconds up to nanoseconds. Although rota- tional and translational motions of anions and cations are intimately coupled and all dynamics are highly collective we may sketch the following rough scenario for all investigated RTILs: The fastest motions are dominated by librations (hindered rotations) of ions in the fluctuating cages formed by their neighbors, superimposed by cage rattling (inter-ionic vibrations). These motions may be modulated by hydrogen bond interactions as, e.g. in EAN. With increasing time librations and cage rattling merge with the dynamics of cage formation and decay, leading to the "constant-loss" region of the spectra, before the $\alpha$ relaxation associated with ion rotation emerges with relaxation times of the order of several tens to a few hundred picoseconds.

Generally, DR and OKE are sensitive simultaneously to anions and cations, albeit with different weight, so that information on specific ions may be difficult to extract. The studied sulfonium and pyrrolidinium RTILs are an example for this. The situation is different for the protic ionic liquids EAN \& PAN, as here DR is specific to cation reorientation whereas the OKE $\alpha$ mode monitors the anion. Similar activation energies for anion and cation rotation suggested cooperativity in their dynamics and the comparison of the overall reorientation of ethylammonium ion (DR) with $\mathrm{N}-\mathrm{H}$ reorientation (time-resolved IR spectroscopy) indicated jump reorientation dictated by the hydrogen-bond network of this RTIL.

For the studied imidazolium RTILs the $\alpha$ relaxation, both in DR and OKE spectra, is dominated by cation reorientation through large-angle jumps. Dipolar anions yield a further DR mode at $\sim 5-10 \mathrm{GHz}$ that is rather weak in amplitude whilst the $\alpha$ mode is enhanced compared to RTILs with nonpolar anions. These observations suggests strong static dipole-dipole correlations of cations and anions if both are dipolar. The most spectacular feature of imidazolium RTILs is the presence of a strong $s u b-\alpha$ peak in the OKE signal indicative for the slow breathing motion of large (possibly $\pi$-stacked) aggregates.

The investigated mixtures of imidazolium salts with polar solvents were found to retain their IL-like character down to surprisingly low RTIL mole fractions of $x \approx 0.2-0.4$ before the dynamics gradually changes to electrolyte-solution like behavior. The latter region was dominated by cation solvation, as well as formation and (at higher $x$ ) redissociation of contact ion pairs. The degree of ion pairing varied with solvent permittivity but was always similar to that of conventional salts with organic cations.

\section{Acknowledgements}

The authors thank all participants of SPP 1191, especially H. Weingärtner and F. Kremer, as well as G. Hefter, C. Schröder and M. Maroncelli for stimulating discussions. Thanks also to W. Kunz for the provision of laboratory facilities at Regensburg. This work was funded by the Deutsche Forschungsgemeinschaft within the priority program 1191 and by the U.K. Engineering and Physical Science Research Council (EPSRC).

[1] P. Wasserscheid, T. Welton, Ionic Liquids in Synthesis, Wiley-VCH, Weinheim, 2003.

[2] H. Weingärtner, Angew. Chem. Int. Ed. 47 (2008) 654.

[3] N. Plechkova, K. R. Seddon, Phys. Chem. Chem. Phys. 37 (2008) 123. 
[4] J. Le Bideau, L. Viau, A. Vioux, Chem. Soc. Rev. 40 (2011) 907.

[5] T. L. Greaves, C. J. Drummond, Chem. Rev. 108 (2008) 206.

[6] H. Niedermeyer, J. P. Hallet, I. J. Villar-Garcia, P. A. Hunt, T. Weltin, Chem. Sov. Rev. 41 (2012) 7780.

[7] R. Buchner, G. Hefter, Phys. Chem. Chem. Phys. 11 (2009) 8954.

[8] C. Wakai, A. Oleinikova, M. Ott, H. Weingärtner, J. Phys. Chem. 109 (2005) 17028.

[9] M.-M. Huang, Y. Jiang, P. Sasisanker, G. W. Driver, H. Weingärtner, J. Chem. Eng. Data 56 (2011) 1494.

[10] G. Giraud, C. M. Gordon, I. R. Dunkin, K. Wynne, J. Chem. Phys. 119 (2003) 464.

[11] D. A. Turton, J. Hunger, G. Hefter, R. Buchner, K. Wynne, J. Chem. Phys. 128 (2008) 161102.

[12] D. A. Turton, J. Hunger, A. Stoppa, G. Hefter, A. Thoman, M. Walther, R. Buchner, K. Wynne, J. Am. Chem. Soc. 131 (2009) 11140.

[13] N. Ito, W. Huang, R. Richert, J. Phys. Chem. B 110 (2006) 4371.

[14] N. Ito, R. Richert, J. Phys. Chem. B 111 (2007) 5016.

[15] J. R. Sangoro, C. Iacob, A. Serghei, C. Friedrich, F. Kremer, Phys. Chem. Chem. Phys. 11 (2009) 913.

[16] C. Krause, J. R. Sangoro, C. Iacob, F. Kremer, J. Phys. Chem. B 114 (2010) 382 .

[17] J. R. Sangoro, F. Kremer, Acc. Chem. Res. 45 (2012) 525.

[18] C. J. F. Böttcher, P. Bordewijk, Theory of electric polarization, Vol.1 \& 2, Elsevier, Amsterdam, 1978.

[19] F. Kremer, A. Schönhals, Broadband Dielectric Spectroscopy, Springer, Berlin, 2003.

[20] J. Barthel, K. Bachhuber, R. Buchner, H. Hetzenauer, M. Kleebauer, Ber. Bunsen-Ges. Phys. Chem. 95 (1991) 853.

[21] J. Barthel, R. Buchner, P. N. Eberspächer, M. Münsterer, J. Stauber, B. Wurm, J. Mol. Liq. 78 (1998) 83.

[22] R. Buchner, J. Barthel, Ber. Bunsen-Ges. Phys. Chem. 101 (1997) 1509.

[23] S. Schrödle, G. Hefter, W. Kunz, R. Buchner, Langmuir 22 (2006) 924.

[24] J. Hunger, Effects of Polar Compounds on the Dynamics and Dielectric Properties of Room-Temperature Ionic Liquids, Ph.D. thesis, Regensburg, 2009.

[25] P. Jepsen, B. M. Fischer, A. Thoman, H. Helm, J. Y. Suh, R. Lopez, R. F. Haglund, Phys. Rev. B 74 (2006) 205103.

[26] M. Walther, B. M. Fischer, A. Ortner, A. Bitzer, A. Thoman, H. Helm, Anal. Bioanal. Chem. 397 (2010) 1009.

[27] D. A. Turton, D. F. Martin, K. Wynne, Phys. Chem. Chem. Phys. 12 (2010) 4191.

[28] D. A. Turton, T. Sonnleitner, A. Ornter, M. Walther, G. Hefter, K. R Seddon, S. Stana, N. Plechkova, R. Buchner, K. Wynne, Faraday Discuss. 154 (2012) 145

[29] R. Buchner, T. Chen, G. Hefter, J. Phys. Chem. B 108 (2004) 2365.

[30] D. A. Turton, K. Wynne, J. Chem. Phys. 128 (2008) 154516.

[31] A. Stoppa, O. Zech, W. Kunz, R. Buchner, J. Chem. Eng. Data 55 (2010) 1768-1773.

[32] S. Shaukat, R. Buchner, J. Chem. Eng. Data 56 (2011) 4944.

[33] S. Schrödle, G. Annat, D. R. MacFarlane, M. Forsyth, R. Buchner, G. Hefter, Chem. Commun. (2006) 1748.

[34] A. Stoppa, J. Hunger, R. Buchner, G. Hefter, A. Thoman, H. Helm, J. Phys. Chem. B 112 (2008) 4854.

[35] J. Hunger, A. Stoppa, S. Schrödle, G. Hefter, R. Buchner, Chem. Phys. Chem. 10 (2009) 723.

[36] J. Hunger, A. Stoppa, R. Buchner, G. Hefter, J. Phys. Chem. B 113 (2009) 9527.

[37] D. A. Turton, J. Hunger, A. Stoppa, G. Hefter, A. Thoman, M. Walther, R. Buchner, K. Wynne, Proc. SPIE 7601 (2010) $76010 H$.

[38] A. Stoppa, R. Buchner, G. Hefter, J. Mol. Liq. 153 (2010) 46.

[39] O. Zech, J. Hunger, J. R. Sangoro, C. Iacob, F. Kremer, W. Kunz, R. Buchner, Phys. Chem. Chem. Phys. 12 (2010) 14341.

[40] Y. Wang, G. Voth, J. Am. Chem. Soc. 127 (2005) 12192.

[41] J. Canongia-Lopes, A. Padua, J. Phys. Chem. B 110 (2006) 3330.

[42] C. Schröder, T. Rudas, O. Steinhauser, J. Chem. Phys 125 (2006) 244506.

[43] A. Triolo, O. Russina, B. Fazio, Chem. Phys. Lett. 457 (2008) 362.

[44] J. Habasaki, K. G. Ngai, J. Chem. Phys. 129 (2008) 194501.

[45] J. R. Sangoro, C. Iacob, A. Serghei, S. Naumov, P. Galvosas, J. Kräger, C. Wespe, F. Bordusa, A. Stoppa, J. Hunger, R. Buchner, F. Kremer, J. Chem. Phys. 128 (2008) 214509.

[46] J. R. Sangoro, C. Iacob, S. Naumov, R. Valiullin, H. Rexhausen,
J. Hunger, R. Buchner, V. Strehmel, J. Kräger, F. Kremer, Soft Matter 7 (2011) 1678.

[47] C. Schröder, T. Sonnleitner, R. Buchner, O. Steinhauser, Phys. Chem. Chem. Phys. 13 (2011) 12240.

[48] Y. Shim, H. Kim, J. Phys. Chem. B 112 (2008) 11028.

[49] B.-R. Hyun, S. V. Dzyuba, R. A. Bartsch, E. L. Quitevis, J. Phys. Chem. A 106 (2002) 7579.

[50] E. W. Castner, J. F. Wishart, H. Shirota, Acc. Chem. Res. 40 (2007) 1217.

[51] E. W. Castner, J. F. Wishart, J. Chem. Phys. 132 (2010) 120901.

[52] T. Ishida, H. Shirota, J. Phys. Chem. B 117 (2013) 1136.

[53] C. Schröder, O. Steinhauser, J. Chem. Phys. 131 (2009) 114504.

[54] H. Weingärtner, A. Knocks, W. Schrader, U. Kaatze, J. Phys. Chem. A 105 (2001) 8646.

[55] K. Fumino, A. Wulf, R. Ludwig, Angew. Chem. Int. Ed. 48 (2009) 5184.

[56] M. Krüger, E. Bründermann, S. Funkner, H. Weingärtner, M. Havenith, J. Chem. Phys. 132 (2010) 101101.

[57] J. Hunger, T. Sonnleitner, L. Liu, R. Buchner, M. Bonn, H. J. Bakker, J. Phys. Chem. Lett. 3 (2012) 3034.

[58] O. Russina, M. Beiner, C. Pappas, M. Russina, V. Arrighi, T. Unruh, C. L. Mullan, C. Hardacre, A. Triolo, J. Phys. Chem. B 113 (2009) 8469.

[59] W. Huang, R. Richert, J. Chem. Phys. 131 (2009) 184501.

[60] A. Stoppa, J. Hunger, G. Hefter, R. Buchner, J. Phys. Chem. B 116 (2012) 7509.

[61] J. Hunger, A. Stoppa, R. Buchner, G. Hefter, J. Chem. Phys. 112 (2008) 12913.

[62] M. Bešter-Rogač, J. Hunger, A. Stoppa, R. Buchner, J. Chem. Eng. Data 56 (2011) 1261.

[63] M. Bešter-Rogač, A. Stoppa, J. Hunger, G. Hefter, R. Buchner, Phys. Chem. Chem. Phys. 13 (2011) 17588. 